\title{
Die notarielle Urkunde im italienisch-deutschen Vergleich: Überlegungen zur Übersetzung von Immobilienkaufverträgen
}

\section{Eva Wiesmann}

Universität Bologna, Italien

wiesmann@sslmit.unibo.it

Notarial documents have some translation-relevant particularities which are strongly associated with the legal culture of the respective country. They are subject to competing influential factors - among others laws and administrative provisions, the facts of the case, form books, notary offices, and the recipient of the document - which determine the content, the specific structure and the language of notarial documents. In addition to the basic parameters of translation, translators should know and tackle the common features and the differences between the notarial documents of the countries concerned in order to produce a professional translation. This paper examines the most important common features and differences between Italian and German real estate sales contracts and presents the implications for translations from Italian into German against the background of the basic parameters of translation.

\section{Einleitung}

Die Übersetzung notarieller Urkunden wurde in der übersetzungswissenschaftlichen Literatur bislang vernachlässigt. Im Allgemeinen wird das Thema nur im Zuge der Auseinandersetzung mit der Urkundenübersetzung angesprochen (z.B. in Fleck, 2003). Lediglich für das Sprachenpaar Spanisch-Deutsch sind mir zwei einschlägige Arbeiten bekannt. ${ }^{1}$ Was dagegen die Übersetzung italienischer notarieller Urkunden ins Deutsche anbelangt, so liegt meines Wissens keinerlei eingehende Auseinandersetzung damit vor. Dem Mangel soll in meiner Monographie zur Sprache des italienischen und des deutschen Notars in Vergangenheit und Gegenwart, die voraussichtlich 2014 erscheinen wird, abgeholfen werden. Dieser Beitrag versteht sich als ein kleiner Schritt in diese Richtung.

Da notarielle Urkunden immer Rechtstexte sind, gilt für sie zunächst einmal, was für die Rechtsübersetzung im Allgemeinen gilt. Es gibt eine Reihe von textinternen und -externen Faktoren, die auf die Übersetzung einen Einfluss haben und die Übersetzungsmethode bedingen (Wiesmann, 2004, S. 82-83). Dabei handelt es sich zum einen um den Text als Produkt einer Rechtskultur, die bei Rechtstexten 
überwiegend, bei notariellen Urkunden stets eine nationale ist. Zum anderen handelt es sich um die Rahmenbedingungen der Übersetzung, nämlich um den Übersetzungszweck, die Empfänger der Übersetzung, die involvierten Rechtsordnungen, das anwendbare Recht und den Status, m.a.W. den rechtlichen Wert der Übersetzung (zur übersetzungsmethodischen Bedeutung des anwendbaren Rechts s. auch Madsen, 1997).

Die notarielle Urkunde, die von Land zu Land verschieden ist und wie alle Rechtstexte im Zuge der Weiterentwicklung des Rechts Änderungen unterliegt, wird dabei ihrerseits durch Faktoren geprägt, nämlich v.a. durch die Rechts- und Verwaltungsvorschriften, den zu regelnden Sachverhalt, die Formularbücher, die Kanzleien und die Empfänger, aber auch durch die Rechtswissenschaft und den zeitgeschichtlichen Hintergrund. Die besagten Faktoren wirken sich in je unterschiedlichem Maße auf den Inhalt der Urkunde, ihren Aufbau und ihre Sprache aus.

Neben den Rahmenbedingungen der Übersetzung müssen Übersetzerinnen und Übersetzer die Unterschiede zwischen den notariellen Urkunden der Länder, aus bzw. in deren Sprache sie übersetzen, kennen und damit professionell umgehen können.

In diesem Beitrag werden am Beispiel des Immobilienkaufvertrags zunächst die wichtigsten Unterschiede herausgearbeitet, die zur heutigen Zeit zwischen italienischen und deutschen notariellen Urkunden bestehen. Im Anschluss daran werden anhand eines Übersetzungsauftrags, wie er in der Praxis tatsächlich vorkommen kann, die Rahmenbedingungen der Übersetzung beschrieben. Daraus werden dann einige Konsequenzen für die Übersetzung eines konkreten italienischen Immobilienkaufvertrags ins Deutsche abgeleitet.

\section{Unterschiede zwischen italienischen und deutschen notariellen Immobilienkaufverträgen}

Italienische und deutsche notarielle Immobilienkaufverträge sind ein Produkt der jeweiligen nationalen Rechtskultur. Dabei sind insbesondere der Aufbau der notariellen Urkunde, die sich daraus ergebenden Inhaltsbestandteile und die sprachliche Form, d.h. die textsortentypischen Formulierungen kulturspezifisch geprägt, während der Inhalt des Rechtsgeschäfts und die damit verbundene Terminologie in dem Maße kulturspezische Unterschiede aufweist, in dem der Immobilienkaufvertrag in Italien und Deutschland unterschiedlich geregelt ist und die beiden Rechtskulturen je andere Begriffe hervorgebracht haben. 


\subsection{Rechts- bzw. Verwaltungsvorschriften und zu regelnder Sachverhalt}

$\mathrm{Zu}$ den wichtigsten Einflussfaktoren gehören die Rechts- und Verwaltungsvorschriften, d.h. die einschlägigen Gesetze sowie in Italien die Durchführungsverordnungen und in Deutschland die Dienstordnungen. Dabei ist zu unterscheiden zwischen Normen, die sich auf den Aufbau der notariellen Urkunde und ihre Sprache beziehen, ${ }^{2}$ und Normen, die in $\mathrm{Zu}-$ sammenhang mit dem zu regelnden Sachverhalt stehen, also den Inhalt des Rechtsgeschäfts betreffen.

Der Aufbau der notariellen Urkunde, und damit auch des notariellen Immobilienkaufvertrags, ist in Italien heute fast vollständig im Notariatsgesetz Nr. 89 von 1913 (LN) verankert. Danach gehören dazu die folgenden Angaben, die - neben der im italienischen Notariatsgesetz nicht genannten Angabe zur Art des Rechtsgeschäfts - die inhaltlichen Bestandteile der notariellen Urkunde bilden:

- Angabe der Nummern von Urkundenrolle und Urkundensammlung;

- $\quad$ Angabe der Rechtsordnung;

- Zeitangabe;

- $\quad$ Ortsangabe;

- $\quad$ Angabe zum Notar;

- $\quad$ Angabe zu den Zeugen (sofern beteiligt);

- $\quad$ Angabe zu den Beteiligten;

- $\quad$ Angabe zur Gewissheit über die Identität der Beteiligten oder die Feststellung ihrer Identität mittels Bürgen;

- $\quad$ Angabe zu den Bürgen (sofern beteiligt);

- $\quad$ Angabe zum Gegenstand der Beurkundung;

- $\quad$ Angabe zu den Anlagen;

- Angabe zur Verlesung der Urkunde und ggf. der Anlagen in Gegenwart der Zeugen;

- $\quad$ Angabe zur Errichtung und zum Umfang der Urkunde;

- Unterschriften der Beteiligten, der Bürgen, des ggf. zugezogenen Dolmetschers, der Zeugen und des Notars.

Neben Bestimmungen zum Aufbau der notariellen Urkunde enthält das italienische Notariatsgesetz auch einige unmittelbare und mittelbare Vorgaben zum Sprachgebrauch, Bestimmungen also, die die Verwendung bestimmter Formulierungen in einer bestimmten Form vorschreiben oder Vorschriften darüber enthalten, wie bestimmte Formulierungen beschaffen sein sollen. Zu den unmittelbaren Vorgaben gehört heute, dass die Angabe der Rechtsordnung mit „REPUBBLICA ITALIANA“ (ITALIENISCHE REPUBLIK) vorgeschrieben ist (Art. $51 \mathrm{LN}$ ). Zu den mittelbaren Vorgaben zählen in der Zeitangabe die Angabe des Datums mit Buchstaben und in der Angabe zum Gegenstand der Beurkundung die 
Vorgabe, dass Datums-, Betrags- und Mengenangaben zumindest beim erstmaligen Vorkommen in der Urkunde in Buchstaben anzugeben sind (Art. $51 \mathrm{LN}$ ).

Abgesehen davon hat das italienische Notariatsgesetz, wie dies bei Gesetzen im Allgemeinen festzustellen ist, auch eine Vorlagenfunktion, was den notariellen Sprachgebrauch anbelangt. So leiten sich beispielsweise aus der Angabe zur Gewissheit (,certezza“; Art. 51 LN) über die Identität der Beteiligten oder die Feststellung (,accertamento“; Art. 51 LN) ihrer Identität mittels Bürgen Formulierungen wie die folgenden aus Avanzini, Iberati und Lovato (2006, S. 4, S. 10) ab:

(1) $[\ldots]$ della cui identità personale sono certo [...]. ([...] deren Identität ich mir gewiss bin [...].)

(2) [...] la cui identità personale mi è accertata dai signori [...], fidefacienti da me conosciuti ed aventi i requisiti di legge [...]. ([...] deren Identität mir bestätigt wurde durch die mir bekannten Bürgen [...], die die gesetzlichen Voraussetzungen erfüllen [...].)

Die gesetzgeberischen Termini „certezza“ und „accertamento“ werden also in den notariellen Urkunden zum Adjektiv „,certo“ bzw. zum Verb „,accertare“, während gleichbedeutend auch das Adjektiv „,sicuro“ und das Verb „,confermare“ hätten verwendet werden können.

$\mathrm{Zu}$ den Normen, die den Inhalt des italienischen Immobilienkaufvertrags anbelangen, gehört in erster Linie das italienische Zivilgesetzbuch. Des Weiteren gehören dazu immobilien- und bau-, städtebau- und verwaltungs- sowie finanz- und steuerrechtliche Normen, die eine Reihe von kulturspezifischen Erklärungen, Gewährleistungen, Selbstverpflichtungen und Anträgen (3) mit sich bringen:

(3) Le spese del presente atto e quelle accessorie e conseguenti, sono a carico della parte acquirente, la quale chiede di poter beneficiare delle agevolazioni fiscali previste dall'articolo 21 della Tabella All. A parte seconda D.P.R. 26 ottobre 1972 n. 633 [...]. (Die Kosten dieser Urkunde und die Neben- und Folgekosten gehen zu Lasten der Käuferpartei, die die Steuerermäßigungen beantragt, wie in Artikel 21 der Tabelle Anhang A Teil II des D.P.R. Nr. 633 vom 26. Oktober 1972 vorgesehen [...].) (Luconi, 2012, S. 122)

In Deutschland ist es heute vor allem das Beurkundungsgesetz (BeurkG) von 1969, das Bestimmungen zum Aufbau der notariellen Urkunde enthält. Weitere für die heutige Zeit relevante Bestimmungen finden sich in der Dienstordnung für Notarinnen und Notare (DONot). 
Insgesamt ergibt sich daraus hinsichtlich der Inhaltsbestandteile das folgende Bild:

- $\quad$ Angabe der Nummer der Urkundenrolle und der Jahreszahl (§ 28 DONot);

- $\quad$ Zeitangabe ( $\$ 9$ BeurkG);

- $\quad$ Angabe zum Notar ( $(9$ BeurkG);

- $\quad$ Ortsangabe $(\S 9$ BeurkG);

- $\quad$ Angabe zu den Beteiligten ( $\$ 9$ BeurkG);

- $\quad$ Angabe darüber, ob der Notar die Beteiligten kennt oder wie er sich Gewissheit über ihre Person verschafft hat (§10 BeurkG);

- $\quad$ Angabe zu den ggf. hinzugezogenen sonstigen Personen (Zeugen, zweiter Notar) (\$29 BeurkG);

- $\quad$ Angabe zum Inhalt des Rechtsgeschäfts, d.h. zum Gegenstand der Beurkundung ( $\$ 9$ BeurkG);

- $\quad$ Angabe darüber, dass der Notar seinen Informationspflichten nachgekommen ist ( $\S 18-21$ BeurkG);

- Angabe betreffend die Vorlesung durch den Notar, die Genehmigung des Inhalts durch die Beteiligten und die eigenhändige Unterzeichnung der Urkunde durch die Beteiligten, die ggf. hinzugezogenen sonstigen Personen und den Notar (§ 13 BeurkG);

- $\quad$ Unterschriften der Beteiligten, der ggf. hinzugezogenen sonstigen Personen und des Notars (versehen mit seiner Amtsbezeichnung) (§13 BeurkG).

Während es auch in den deutschen notariellen Urkunden, wenngleich in den einschlägigen Normen nicht eigens erwähnt, eine Angabe zur Art des Rechtsgeschäfts und eine Angabe zu den Anlagen gibt, fehlen dort die Angabe der Rechtsordnung und die Angabe zur Errichtung und zum Umfang der Urkunde völlig. Die Reihenfolge der Inhaltsbestandteile ist dagegen in Italien und in Deutschland im Wesentlichen die gleiche.

Anders verhält es sich bei den Vorgaben zum Sprachgebrauch, die in Deutschland heute in geringerem Umfang als in Italien zu finden sind und nur mittelbare Vorgaben darstellen. So heißt es in den Normen insbesondere, wichtige Zahlen seien in Ziffern und Buchstaben zu schreiben ( $\$ 28$ DONot). Für die Vorlagenfunktion für den notariellen Sprachgebrauch gilt dagegen wieder Ähnliches wie in Italien. Ein Beispiel dafür ist das aus dem im Beurkundungsgesetz (§ 9) verwendeten Terminus „Verhandlung“ abgeleitete Partizip „verhandelt“ als eine Formulierungsmöglichkeit für die Orts- und die Zeitangabe in der deutschen notariellen Urkunde:

(4) Verhandelt am [...] in [...] / Vor mir, / [...] / Notar in [...] erschienen heute in meinen Amtsräumen: / [...]. (Münchener Vertragshandbuch, 1998, 4/1.I.32) 
Eine andere Formulierungsmöglichkeit für die betreffenden Angaben ist daneben:

(5) Heute, am [...], sind vor mir, / [...] / Notar mit dem Amtssitz in [...], in der Geschäftsstelle [...] gleichzeitig anwesend: / [...]. (Text Berufspraxis)

Wie beim italienischen Immobilienkaufvertrag, so wirken sich auch auf den Inhalt des deutschen eine Reihe von Normen aus. Im Gegensatz zu Italien wird aber auf weniger Normen explizit Bezug genommen, so in erster Linie auf das Bürgerliche Gesetzbuch (BGB) (6) und in zweiter Linie auf immobilien-, bau-, städtebau- und steuerrechtliche Normen.

(6) Der Verkäufer bewilligt und der Käufer beantragt zur Sicherung des Anspruchs des Käufers auf Verschaffung des Eigentums die Eintragung einer Vormerkung gemäß $\S 883$ BGB in das Grundbuch. (Text Berufspraxis)

Damit können wiederum kulturspezifische Erklärungen, Gewährleistungen, Selbstverpflichtungen, Anträge und - wie das Beispiel zeigt auch Bewilligungen verbunden sein.

Aus den unterschiedlichen Regelungen des Immobilienkaufvertrags in Italien und in Deutschland ergeben sich wichtige rechtliche Unterschiede, Differenzen in der Binnengliederung der Angabe zum Inhalt des Rechtsgeschäfts und in der inhaltlichen und der sprachlichen Ausgestaltung der Vertragsbestimmungen sowie begriffliche Besonderheiten, die in der Terminologie zum Ausdruck kommen.

$\mathrm{Zu}$ den wichtigsten rechtlichen Unterschieden gehört, dass der Rechtsübergang in Italien im Zeitpunkt der Einigung erfolgt, in Deutschland dagegen über die beim Immobilienkaufvertrag „Auflassung“ genannte Einigung hinaus die Eintragung des Rechtsübergangs im Grundbuch erforderlich ist.

Was die Binnengliederung der Angabe zum Inhalt des Rechtsgeschäfts anbelangt, so gibt es Vertragsbestandteile, die entweder nur in Italien oder nur in Deutschland vorkommen. Ein Beispiel dafür sind die in italienischen Immobilienkaufverträgen erforderlichen Angaben zur Baugenehmigung oder zur nachträglichen Genehmigung bzw. zu dem darauf gerichteten Antrag, deren Fehlen die Nichtigkeit des Vertrags zur Folge hat (Kindler, 2000, S. 266). Ein anderes Beispiel sind die in deutschen Immobilienkaufverträgen stets vorhandenen Angaben dazu, wer von der Urkunde jeweils eine beglaubigte und wer davon eine einfache Abschrift erhält.

Die Unterschiede in der inhaltlichen und der sprachlichen Ausgestaltung von Vertragsbestimmungen betreffen beispielsweise den Gegenstand des Immobilienkaufvertrags. Während in deutschen Immobilienkaufverträgen in tabellarischer Form im Wesentlichen die 
Grundbucheintragungen angegeben werden, enthalten die italienischen Immobilienkaufverträge über die entsprechenden Katasterangaben hinaus eine in einen komplexen Satz eingebundene Angabe zur Art, Lage und Größe des Grundstücks (Kindler, 2000, S. 271).

$\mathrm{Zu}$ den für die Übersetzung italienischer Immobilienkaufverträge ins Deutsche wichtigsten begrifflich-terminologischen Besonderheiten schließlich zählen: ${ }^{3}$

- $\quad$ die für fast ganz Italien geltende Unterscheidung zwischen den Begriffen „registro immobiliare“ (Immobilienregister; Verzeichnis der Rechtsverhältnisse von Grundstücken; deklaratorische Wirkung der Eintragung des Immobilienerwerbs) und „catasto“ (Kataster; Verzeichnis der Grundstücke unter Angabe ihrer Ertragsmöglichkeiten und ihrer Eigentümer), die nur bedingt der begrifflichen Unterscheidung zwischen „Grundbuch“ (Verzeichnis der Grundstücke und der diesbezüglichen Rechtsverhältnisse; konstitutive Wirkung der Eintragung des Immobilienerwerbs) und „Liegenschaftskataster“ (Verzeichnis der Grundflächen) entspricht;

- $\quad$ die - wiederum für fast ganz Italien geltende - begriffliche Unterscheidung zwischen „trascrizione“ (Eintragung beim Amt für Liegenschaften; die Eintragung des Immobilienkaufvertrags erfolgt aus Publizitätsgründen), ,registrazione“ (Eintragung beim Amt für Finanzen; die Eintragung des Immobilienkaufvertrags erfolgt aus steuerlichen Gründen) und „,annotamento“ bzw. „annotazione“ (Änderungseintragung; die Eintragung betrifft Änderungen in Bezug auf bereits erfolgte Eintragungen von Immobilienkaufverträgen beim Amt für Liegenschaften), für die es in Deutschland keine Entsprechung gibt;

- $\quad$ die damit in Zusammenhang stehende Unterscheidung zwischen den Behörden „Agenzia delle Entrate“ (Amt für Finanzen) und „Agenzia del Territorio“ (Amt für Liegenschaften), wobei Letztere seit dem 1.12.2012 Teil der Ersteren ist und in Deutschland kein Pendant hat;

- die in Deutschland entsprechungslose „evizione“ (Eviktion), die den Käufer, der vom Nichteigentümer gekauft hat, berechtigt, gegen den Verkäufer vorzugehen, wenn er in seinem Besitz gestört ist oder gestört zu werden droht (Kindler, 2000, S. 271);

- die Begriffe, die in Zusammenhang mit den in Italien erforderlichen Angaben zur Rechtmäßigkeit der Bauausführung stehen: neben dem Begriff ,permesso di costruire“ (Baugenehmigung) die Begriffe „denuncia di inizio / fine attività“ (Anzeige über den Beginn / den Abschluss der Bauarbeiten) sowie „,ertificato di conformità ed agibilità“ (bauliche Übereinstimmungs- und Benutzbarkeitsbescheinigung; Bescheinigung darüber, dass der Bau wie genehmigt ausgeführt wurde); während der Begriff „Baugenehmigung“ auch in deutschen Immobilien- 
kaufverträgen vorkommt, gibt es in Deutschland zwar Bescheinigungen über den Beginn bzw. das Ende von Bauarbeiten, sie sind jedoch nicht typisch für deutsche Immobilienkaufverträge, in denen auch nichts mit der baulichen Übereinstimmungs- und Benutzbarkeitsbescheinigung Vergleichbares vorkommt;

- $\quad$ die bei der Inanspruchnahme von Steuerermäßigungen in Italien relevante Unterscheidung zwischen „prima casa“ (Erstwohnung; zu Wohnzwecken dienende Immobilie, die zum Zeitpunkt des Erwerbs die einzige im Eigentum des Käufers ist und in der der Käufer seinen Wohnsitz begründet) und „abitazione principale“ (Hauptwohnsitz; Immobilie, in der der Wohnsitz begründet wird und die den Ort des gewöhnlichen Aufenthalts darstellt), für die es in Deutschland keine Entsprechung gibt;

- bei Immobilien schließlich, bei denen nicht nur Voll-, sondern auch Miteigentum an gemeinschaftlichen Teilen erworben wird, der gleichfalls typisch italienische Begriff „beni comuni non censibili“" (gemeinschaftliche Teile ohne Katasterertrag; nach dem Katasterertrag richtet sich in Italien die jährliche Besteuerung des Volleigentums an Immobilien, während das Miteigentum an gemeinschaftlichen Teilen von der Besteuerung ausgenommen ist).

\subsection{Formularbücher und Kanzleien sowie Empfänger}

Weitere wichtige Einflussfaktoren sind in Italien wie in Deutschland die Formularbücher und die Kanzleien.

Formularbücher sind in Buchform veröffentlichte Sammlungen von Mustertexten für alle Formen der Rechtsanwendung und der Rechtspraxis, so auch für die notarielle Praxis. Sie haben eine lange, bis ins Mittelalter, ja bis ins Altertum zurückreichende Tradition und dokumentieren für Notare Folgendes:

- $\quad$ die gesetzlich vorgeschriebenen inhaltlichen Bestandteile von notariellen Urkunden einschließlich der rechtlichen Gestaltungsmöglichkeiten für die jeweiligen Rechtsgeschäfte;

- $\quad$ die gesetzlichen Vorgaben für die sprachliche Formulierung;

- die Konventionen der sprachlichen Formulierung, die z.T. auf gesetzlichen Formulierungen beruhen.

Kanzleien arbeiten heute zwar mit Formularbüchern, sie erstellen jedoch auch ihre eigenen Mustertextsammlungen, passen sie der Fortentwicklung des Rechts an und geben sie an ihre Nachfolger weiter. Insofern wirken auch sie sich in inhaltlicher wie in sprachlicher Hinsicht auf die notarielle Urkunde aus.

Formularbücher und Mustertextsammlungen von Kanzleien sind dabei ihrerseits Einflüssen ausgesetzt. Was den Inhalt, nämlich die recht- 
lichen Gestaltungsmöglichkeiten anbelangt, so sind hierfür zum einen die einschlägigen Normen maßgeblich und zum anderen die rechtswissenschaftliche Auseinandersetzung damit, die sich in den notariellen Urkunden sowohl mittel- als auch unmittelbar niederschlägt. In sprachlicher Hinsicht kommt neben den Normen und der rechtswissenschaftlichen Auseinandersetzung mit notariellen Urkunden auch der zeitgeschichtliche Hintergrund in Frage, und zwar insofern, als Formulierungen, die früher gebräuchlich waren, heute als überholt erachtet werden können, ohne dass die einschlägigen Normen dazu eine Regelung vorsehen (Wiesmann, 2013). Auch dies kann sich sowohl mittel- als auch unmittelbar auf die notariellen Urkunden auswirken.

Im Vordergrund stehen im Folgenden die textsortentypischen Formulierungen von heutigen notariellen Urkunden, die Ausdruck des juristischen Stils im Allgemeinen und des notariellen Stils im Besonderen sind und durch Formularbücher und Mustertextsammlungen von Kanzleien maßgeblich mitgeprägt werden. Diesbezüglich sollen die wichtigsten Unterschiede zwischen italienischen und deutschen Immobilienkaufverträgen unter Berücksichtigung der Empfänger herausgearbeitet werden.

Die Empfänger von notariellen Urkunden sind in erster Linie die am Rechtsgeschäft Beteiligten, beim Immobilienkaufvertrag also die Vertragsparteien, die eine beglaubigte Abschrift bekommen. Weitere Empfänger sind der Notar, bei dem die Urschrift verbleibt, im Falle eines Darlehens die Bank, in Italien darüber hinaus das Amt für Finanzen und bis vor kurzem auch das Amt für Liegenschaften, in Deutschland das Grundbuchamt, das Finanzamt und der Gutachterausschuss. Im Streitfall kommen in beiden Ländern als Empfänger Rechtsanwälte und Richter in Frage. Da die primären Empfänger in der Regel Laien sind, wäre zu erwarten, dass die Notare ihnen sprachlich im Rahmen des Möglichen entgegenkommen. Wie zu sehen sein wird, ist das aber v.a. bei den italienischen notariellen Urkunden nicht der Fall, die als eigentliche Empfänger Juristen und nicht Laien erkennen lassen und auch an das Verständnis der Übersetzerinnen und Übersetzer höhere Ansprüche stellen. ${ }^{4}$

Erste Unterschiede zwischen italienischen und deutschen notariellen Urkunden ergeben sich bereits bei der Formulierung der in beiden gleichermaßen vorhandenen Zeitangabe, Ortsangabe und Angabe zum Notar:

(7) L'anno duemilasette, il giorno [...] del mese di febbraio in [...], nel mio studio in [...] n. [...]. / [...] FEBBRAIO 2007 / Innanzi a me dott. [...] Notaio in [...], iscritto presso il Collegio Notarile dei Distretti Riuniti di [...] e [...]; senza l'assistenza dei testimoni per avervi i comparenti rinunziato tra loro d'accordo e con il mio consenso; / Sono presenti i Signori: / [...]. (Luconi, 2012, S. 109) (Übersetzung s. Beisp. 18) 
(8) Heute, den $[\ldots] /-[\ldots]-/$ erschienen vor mir, / [...], / Notar in [...], an der Geschäftsstelle in [...] / [...]. (Text Berufspraxis)

Die Unterschiede betreffen insbesondere die Konventionen der Datumsangabe (,L'anno duemilasette, il giorno [...] del mese di febbraio“ vs. „Heute, den [...]“), die Zahl (eine temporale, zwei lokale und eine modale vs. eine temporale Angabe) und die syntaktische Komplexität (,senza l'assistenza dei testimoni per avervi i comparenti rinunziato tra loro d'accordo e con il mio consenso") der Angaben vor dem Verb sowie lexikalische und orthographische Besonderheiten (wie das literatursprachliche ,innanzi“ und die weniger gebräuchliche Schreibweise von „,rinunciare“ mit ,z“ statt mit „,c“), die bei den Vertragsparteien sowie bei den Übersetzerinnen und Übersetzern zumindest den Verständnisaufwand erhöhen.

$\mathrm{Zu}$ den archaisch anmutenden Stilmerkmalen italienischer notarieller Urkunden, die nicht im Dienst der Verständlichkeit stehen (ausführlich Mortara Garavelli, 2006, S. 88-90) und zu denen es in deutschen Urkunden kein Pendant gibt, gehören die Verwendung von „esso“ als adjektisch gebrauchtes Demonstrativpronomen in Verbindung mit einem bereits genannten Substantiv:

(9) [...] provvedimenti che essa Società venditrice, come sopra rappresentata, si impegna comunque ad ottenere [...]. ([...] verpflichtet sich die verkaufende Gesellschaft, vertreten wie vor, die oben genannten Bescheinigungen [...] zu erlangen [...].) (Luconi, 2012, S. 122)

Weitere Unterschiede betreffen den sprachlichen Ausdruck der Einigung der Vertragsparteien, der für italienische Verträge im Allgemeinen kennzeichnend ist, in notariellen Immobilienkaufverträgen aber - wie das folgende Beispiel zeigt - darüber hinaus in eine Erklärung eingebunden wird:

(10) La Società [...] dichiara di vendere alla Signora [...], che dichiara di acquistare, il seguente immobile: / [...]. (Luconi, 2012, S. 110-111) (Übersetzung s. Beisp. 20)

In deutschen Verträgen, und auch in notariellen Immobilienkaufverträgen, ist ein vergleichbarer sprachlicher Ausdruck der Einigung, der von einigen italienischen Rechtswissenschaftlern, ebenso wie die häufige Wiederholung von ,dichiarare“, als überflüssig erachtet wird (La Porta, 2006, S. 74; Marmocchi, 2006, S. 26) und insofern den Verständnisaufwand unnötig erhöht, nicht üblich und auch das Verb „,erklären“ wird - wie am folgenden Beispiel zu sehen ist - dort an übergeordneter Stelle genannt: 
(11) Die Beteiligten erklären folgendes: / I. Grundbuchstand / [...] / II. Verkauf / [...] / - nachstehend als „Verkäufer“ bezeichnet - / verkauft mit allen damit verbundenen Rechten und Pflichten und den gesetzlichen Bestandteilen / an / [...] / - nachstehend als „Käufer“ bezeichnet - / zum Alleineigentum / den in Abschnitt I. näher beschriebenen Grundbesitz samt den aufstehenden Gebäulichkeiten / - nachstehend als „Kaufobjekt“ bezeichnet -. / [...]. (Text Berufspraxis; Hervorhebung im Original)

Auch hinsichtlich der Verwendung von Paarformeln, die als redundant zu betrachten sind und mit Blick auf den Verständnisaufwand insbesondere von Übersetzerinnen und Übersetzern, die die Redundanz schließlich erst einmal erkennen müssen, eingespart werden könnten (ausführlich Mortara Garavelli, 2006, S. 90-92), bestehen Unterschiede zwischen italienischen und deutschen Verträgen im Allgemeinen und notariellen Immobilienkaufverträgen im Besonderen. So stehen z.B. einem italienischen Ausdruck wie ,[...] convengono e stipulano quanto segue“ in deutschen Verträgen der Ausdruck ,,vereinbaren [...] Folgendes“ und in deutschen notariellen Immobilienkaufverträgen der Ausdruck ,erkären [...] Folgendes" gegenüber.

Ein typisches, das Verständnis durch die Vertragsparteien und durch die Übersetzerinnen und Übersetzer erschwerendes Merkmal italienischer Immobilienkaufverträge ist die Opazität von Formulierungen wie der folgenden, wo mit „ora per allora“ gemeint ist, dass die abschließende Quittung rückwirkend auszustellen ist:

(12) Stante quanto sopra la Società venditrice, come sopra rappresentata, rilascia ora per allora definitiva quietanza di saldo ad avvenuto pagamento dell'intero prezzo. (Angesichts dessen stellt die verkaufende Gesellschaft, vertreten wie vor, hierüber nach erfolgter Zahlung des gesamten Kaufpreises rückwirkend eine abschließende Quittung aus.) (Luconi, 2012, S. 114)

Ein weiteres, das Verständnis italienischer Immobilienkaufverträge erschwerendes Merkmal ist die extreme Komplexität der Syntax, die in deutschen Immobilienkaufverträgen in geringerem Maße gegeben ist:

(13) I beni negoziati vengono trasferiti nella loro attuale consistenza di fatto e di diritto, con ogni accessione, pertinenza, diritto, onere e servitù attive e passive se e come in atto legalmente esistenti ed in particolare con la servitù di elettrodotto in conduttori aerei e cavo sotterraneo a favore dell',ENEL Distribuzione S.p.A.“, con sede in Roma, costituita con scrittura privata autenticata nelle firme da me Notaio in data [...] 2006 repertorio n. [...] ed in data [...] 2006 repertorio $\mathrm{n}$. [...], registrata a [...] in data [...] 2007 al n. [...], trascritta presso l'Agenzia del Territorio - Ufficio Provinciale di 
[...] in data [...] 2007 all'articolo [...], nonché con tutti i patti, obblighi e servitù contenuti o richiamati nell'atto ai miei rogiti in data [...] 2003, repertorio n. [...] di cui appresso, e con quelli derivanti dall'uso delle parti comuni del fabbricato come per legge e dal regolamento di condominio con relative tabelle millesimali, che disciplina l'uso, la comproprietà e l'amministrazione dell'intero edificio, che è stato allegato al mio precedente atto in data odierna repertorio n. [...] in corso di registrazione $\mathrm{e}$ trascrizione perché nei termini, e che la parte acquirente dichiara di ben conoscere ed accettare, obbligandosi ad osservare quanto in esso previsto e convenuto. (Luconi, 2012, S. 115-116) (Übersetzung s. Beisp. 19)

Außerdem weisen italienische notarielle Urkunden auch eine Reihe von syntaktischen Besonderheiten auf, die Mortara Garavelli (2006, S. 93) als „stereotipi sintattici“ (syntaktische Stereotype) bezeichnet. Dazu gehören u.a. die Abweichungen von der normalen Stellung der Satzglieder (s. Beisp. 7) und die bevorzugte Verwendung von Partizipien (14) und Gerundien (15) statt von Nebensätzen, wobei Partizipien allerdings auch für deutsche notarielle Urkunden typisch sind (16). Gleiches gilt für den Nominalstil und die unpersönlichen Formulierungen, die ein allgemeines Merkmal der italienischen und der deutschen Rechtssprache darstellen.

(14) [...]; il tutto confinante in complesso con parti comuni [...]. ([...]. Dies alles grenzt insgesamt an das Miteigentum am Gesamtbau an [...].) (Luconi, 2012, S. 111)

(15) $[\ldots]$ esonerando il Signor Direttore dell'Agenzia del Territorio [...] da ogni responsabilità al riguardo. ([...] und befreit den Direktor des Amts für Liegenschaften [...] von jeglicher Haftung diesbezüglich.) (Luconi, 2012, S. 114)

(16) Diese Rechte sind dem Käufer bekannt und sie werden von ihm einschließlich der aus ihnen erwachsenden persönlichen Verpflichtungen mit schuldbefreiender Wirkung übernommen. (Text Berufspraxis)

Auffällig ist schließlich noch, dass in italienischen Immobilienkaufverträgen keinerlei Termini erklärt werden, während das in deutschen Immobilienkaufverträgen mitunter der Fall ist, wie das folgende Beispiel zeigt:

(17) III. Auflassung, Vormerkung / 1. / Die Vertragsteile sind über den Eigentumsübergang auf den Käufer einig (Auflassung). (Text Berufspraxis; Hervorhebung im Original) 


\section{3. Übersetzung italienischer notarieller Immobilienkaufverträge ins Deutsche}

Gegenstand der Übersetzung, um die es im Folgenden geht, ist ein Immobilienkaufvertrag aus dem Jahr 2007, der von einem italienischen Notar errichtet wurde. Es handelt sich um einen Vertrag zwischen einer italienischen Gesellschaft mit beschränkter Haftung und einer deutschen Staatsbürgerin, die die Übersetzung benötigt, um sich eine genaue Vorstellung vom Vertragsinhalt zu machen. Die Übersetzung wird also zum Zweck der Verständnishilfe benötigt und der Empfänger ist die deutsche Vertragspartei. Die involvierten Rechtsordnungen sind insofern zwei, als die zu übersetzende italienische Urkunde nicht für einen italienischen Staatsbürger aus Südtirol, sondern für eine deutsche Staatsbürgerin aus der Bundesrepublik zu übersetzen ist, die zwar keine Juristin ist, sich aber doch über Schule, Universität und Medien mit der deutschen Rechtskultur vertraut gemacht hat. Das auf den Immobilienkaufvertrag anwendbare Recht ist das italienische, d.h. für seine Auslegung ist das italienische Recht maßgeblich. Die Übersetzung kann im Streitfall bei der Auslegung allenfalls hilfsweise herangezogen werden, hat aber im Gegensatz zur italienischen Urkunde keinen rechtlich relevanten Status.

Die Konsequenzen aus der Gesamtheit der textinternen und -externen Faktoren sind, dass zielkulturelle Elemente nur bei ausreichender Entsprechung hinsichtlich des Inhalts bzw. der Funktion verwendet werden können, die Übersetzung aber ansonsten ausgangskulturorientiert $\mathrm{zu}$ erfolgen hat. Hierbei sind folgende Unterscheidungen zu treffen.

Was den Aufbau der notariellen Urkunde, die Reihenfolge der daran gebundenen Inhaltsbestandteile sowie die Binnengliederung der Angabe zum Inhalt des Rechtsgeschäfts anbelangt, so muss sich die Übersetzung am Ausgangstext orientieren. Syntaktische Anpassungen, die im Dienst der Verständlichkeit für den zielkulturellen Empfänger stehen, sind nur im Rahmen der vorgegebenen Textstruktur möglich. Ein Beispiel hierfür ist die Voranstellung des Hilfsverbs in der Übersetzung von Beisp. 7:

(18) Am [...] Februar zweitausendsieben sind in [...], in meiner Kanzlei in [...] Nr. [...], / [...] FEBRUAR 2007 / vor mir Dott. [...], Notar in [...], eingetragen im Verzeichnis der zusammengelegten Notariatsbezirke von [...] und [...], ohne Hinzuziehung von Zeugen, auf die die Erschienenen einvernehmlich und mit meiner Zustimmung verzichtet haben, / anwesend: [...] (Luconi, 2012, S. 109)

Auch bei besonders komplexen Satzstrukturen sind syntaktische Anpassungen möglich, aber nur dann, wenn Sätze getrennt werden können, ohne dass - wie bei der Übersetzung von Beisp. 13 - der Sinnzusammenhang verloren geht: 
(19) Die vertragsgegenständlichen Sachen werden in ihrem heutigen tatsächlichen und rechtlichen Zustand übertragen, und zwar mit sämtlichem Zuwachs und Zubehör sowie mit allen Rechten, Lasten und Dienstbarkeiten zu ihren Gunsten und Lasten, wenn und wie sie rechtmäßig vorhanden sind, insbesondere mit der Stromleitungsdienstbarkeit in Form von oberirdischen Leitungen und unterirdischen Kabeln zu Gunsten von "ENEL Distribuzione S.p.A.", mit Sitz in Rom. Die betreffende Stromdienstbarkeit wurde mit Privaturkunde begründet, die von mir, Notar, am [...] 2006, Urkundenrolle Nr. [...], und am [...] 2006, Urkundenrolle Nr. [...], unterschriftsbeglaubigt wurde, eingetragen am [...] 2007 beim Finanzamt in [...] unter der Nr. [...] und beim Amt für Liegenschaften - Provinzialamt [...] am [...] 2007 unter dem Artikel [...]. Außerdem werden die vertragsgegenständlichen Sachen mit allen Abmachungen, Pflichten und Dienstbarkeiten übertragen, die in der von mir, Notar, am [...] 2003 errichteten, unten genannten Urkunde, Urkundenrolle Nr. [...], enthalten sind bzw. auf die dort verwiesen wird, sowie mit allen Abmachungen, Pflichten und Dienstbarkeiten, die sich aus dem Gebrauch der gemeinschaftlichen Teile des Gebäudes gemäß dem Gesetz und aus der mit Tausendsteltabelle versehenen Gemeinschaftsordnung ergeben, die den Gebrauch, das Miteigentum und die Verwaltung des ganzen Gebäudes regelt und die der von mir, Notar, am heutigen Tag vorher errichteten Urkunde, Urkundenrolle Nr. [...], die sich in der Phase der noch fristgemäßen Eintragung beim Finanzamt und beim Amt für Liegenschaften befindet, als Anlage beigefügt worden ist und bezüglich derer die Käuferpartei erklärt, dass sie sie gut kennt und annimmt, und sich verpflichtet, ihre Vorschriften und Bestimmungen zu beachten. (Luconi, 2012, S. 115-116)

Eine zielkulturelle Orientierung sollte insbesondere bei Konventionen wie denen der Datumsangabe, bei Paarformeln und sonstigen Redundanzen sowie bei Archaismen erfolgen, da es hierzu jeweils Entsprechungen gibt, nämlich zum einen gleichwertige Datumskonventionen und zum anderen gleichwertige nicht redundante bzw. nicht archaische Ausdrücke. Gleiches gilt für diejenigen syntaktischen Stereotype von italienischen notariellen Urkunden, die auch in deutschen Urkunden stilbildend sind.

Die Opazität sollte bei der Übersetzung aus dem Italienischen ins Deutsche mit Blick auf die Verständlichkeit möglichst beseitigt werden. Zwei treffende Beispiele hierfür sind die Übersetzungen der in Beisp. 13 vorkommenden Ausdrücke „,nell'atto ai miei rogiti“ und ,in corso di registrazione e trascrizione perché nei termini“ mit in der von mir, Notar, [...] errichteten [...] Urkunde bzw. mit die sich in der Phase der noch fristgemäßen Eintragung beim Finanzamt und beim Amt für Liegenschaften befindet, wie in Beisp. 19 vorgeschlagen. 
Beim sprachlichen Ausdruck der Einigung der Vertragsparteien ist eine Orientierung am Ausgangstext möglich, wie die Übersetzung von Beisp. 10 zeigt:

Die Gesellschaft [...] erklärt, [...] die folgende Immobilie an Frau [...] zu verkaufen, die erklärt, die folgende Immobilie zu erwerben, und zwar: / [...]. (Luconi, 2012, S. 110-111)

Möglich ist aber auch ein Verzicht auf das Verb „erklären“ und den Relativsatz (Die Gesellschaft [...] verkauft die folgende Immobilie an Frau $[\ldots])$ und damit eine zielkulturelle Orientierung.

Was schließlich die Terminologie (s. 2.1.) anbelangt, so kann bei annähernder Äquivalenz (,permesso di costruire“ / Baugenehmigung) stets zielkulturorientiert übersetzt werden, während die Ausgangskulturorientierung bei Nulläquivalenz oder zu geringer Teiläquivalenz geboten ist (zum Äquivalenzbegriff s. u.a. Šarčević, 1997). Ein Beispiel hierfür ist die Übersetzung in einer Weise, die das Fremde entweder durchscheinen lässt (Amt für Finanzen statt der in Luconi 2012 verwendeten Übersetzung Finanzamt) oder es erklärt (Immobilienregister, Amt für Liegenschaften, Eintragung beim Amt für Liegenschaften, Eintragung beim Amt für Finanzen, Änderungseintragung, bauliche Übereinstimmungs- und Benutzbarkeitsbescheinigung, Anzeige über den Beginn / den Abschluss der Bauarbeiten, gemeinschaftliche Teile ohne Katasterertrag) oder aber auf lateinische Ausdrücke (Eviktion) zurückgreift. Nicht ganz unproblematisch sind die Fälle von zu geringer Teiläquivalenz, die dennoch als Übersetzungen voneinander verwendet werden (,prima casa“ / Erstwohnung, ,abitazione principale“ / Hauptwohnsitz, ,,catasto“ / Kataster), weil sie sich als Übersetzungen so eingebürgert haben. Hier kann u.U. eine zusätzliche Erklärung erforderlich sein.

Der Rahmen, in dem bei der Rechtsübersetzung eine zielkulturelle Orientierung möglich und eine ausgangskulturelle Orientierung geboten ist, wird, so lässt sich zusammenfassend festhalten, durch die besondere Konstellation von textinternen und -externen Faktoren abgesteckt, die bei jedem Übersetzungsauftrag anders gegeben ist. Eine zentrale Stellung nimmt darunter bei der Übersetzung von Immobilienkaufverträgen die notarielle Urkunde (textinterner Faktor) als Produkt einer stets nationalen Rechtskultur ein, deren Kenntnis mit Bezug auf die Ausgangs- wie auf die Zielrechtsordnung Übersetzerinnen und Übersetzern das Verständnis des zu übersetzenden Texts ermöglicht und sie die für die Übersetzung relevanten Gemeinsamkeiten und Unterschiede erkennen lässt. Der übersetzungsmethodische Umgang damit verlangt dann die Berücksichtigung sämtlicher Rahmenbedingungen (textexterne Faktoren). 


\section{Literaturverzeichnis}

Avanzini, A., Iberati, L., \& Lovato, A. (2006). Formulario degli atti notarili. Torino: Utet.

Fleck, K. E. W. (2003). Urkundenübersetzung. In M. Snell-Hornby, H. G. Hönig, P. Kußmaul, \& P. A. Schmitt (Hgg.), Handbuch Translation (S. 230-234). Tübingen: Stauffenburg.

Holl, I. (2011). El documento notarial en España y en Alemania: Un estudio contrastivo como ejercicio previo a la traducción. In S. Roiss, C. Fortea Gil, A. Recio Ariza, B. Santana López, P. Zimmermann, \& I. Holl (Hgg.), En las vertientes de la traducción e interpretación del/al alemán (S. 407-430). Berlin: Frank \& Timme.

Kindler, P. (2000). Immobilienerwerb in Italien. MittBayNot, 4, 265-279.

La Porta, U. (2006). Il linguaggio nell'attività notarile. In E. Marmocchi (Hg.), L'atto pubblico fra tecniche di redazione e forme di comunicazione (S. 69-82). Mailand: Giuffrè.

Luconi, M. (2012). Il contratto di compravendita immobiliare in Italia e in Germania: Personal Knowledge Management e traduzione. Tesi di laurea Università di Bologna.

Madsen, D. (1997). A model for translation of legal texts. In M. Snell-Hornby, Z. Jettmarová, \& K. Kaindl (Hgg.), Translation as intercultural communication (S. 291-299). Amsterdam: John Benjamins.

Marmocchi, E. (2006). L'atto pubblico e il suo ordine. In E. Marmocchi (Hg.), L'atto pubblico fra tecniche di redazione e forme di comunicazione (S. 19-36). Mailand: Giuffrè.

Mortara Garavelli, B. (2006). L'occhiale del linguista su testi notarili. In E. Marmocchi (Hg.), L'atto pubblico fra tecniche di redazione e forme di comunicazione. (S. 83-98). Mailand: Giuffrè.

Münchener Vertragshandbuch (1998). Bd. 4/1 Bürgerliches Recht. München: Beck.

Šarčević, S. (1997). New approach to legal translation. Den Haag: Kluwer Law International.

Stengel-Hauptvogel, I. (1997). Juristisches Übersetzen Spanisch-Deutsch: Immobilienkaufverträge. Tübingen: Narr.

Wiesmann, E. (2004). Rechtsübersetzung und Hilfsmittel zur Translation: Wissenschaftliche Grundlagen und computergestützte Umsetzung eines lexikographischen Konzepts. Tübingen: Narr.

Wiesmann, E. (2012). Die Sprache des deutschen Notars gestern und heute: Dimensionen der Verständlichkeit. In S. Moraldo (Hg.), Sprachenpolitik und Rechtssprache: Methodische Ansätze und Einzelanalysen (S. 153-190). Frankfurt: Peter Lang.

Wiesmann, E. (2013). Der notarielle Sprachgebrauch und seine Beeinflussung durch Normen: Ein Vergleich zwischen Italien und Deutschland. In L. Sergo, U. Wienen, \& V. Atayan (Hgg.), Fachsprache(n) in der Romania: Entwicklung, Verwendung, Übersetzung (S. 421-441). Berlin: Frank \& Timme. 
1 Hierbei handelt es sich um die auf eine übersetzungstheoretische Diskussion bedauerlicherweise verzichtende Monographie von Stengel-Hauptvogel, 1997, zur Übersetzung spanischer Immobilienkaufverträge ins Deutsche und den Aufsatz von Holl, 2011, in dem ein typologischer Vergleich spanischer und deutscher notarieller Urkunden vorgeschlagen wird.

2 In Wiesmann (2013) wird der diesbezügliche Einfluss auf die notarielle Urkunde in diachronischer Perspektive untersucht. Dieser Beitrag ist dagegen synchronisch ausgerichtet.

3 Die Übersetzungen der italienischen Termini stammen überwiegend aus der Masterarbeit von Luconi (2012), die u.a. eine Musterübersetzung eines real existierenden italienischen Immobilienkaufvertrags ins Deutsche angefertigt hat.

4 In Bezug auf deutsche Immobilienkaufverträge wurde in Wiesmann (2012) herausgearbeitet, inwieweit sich deren Verständlichkeit im Lauf der Zeit verbessert hat. 